\title{
Mitochondrial Alteration: A Major Player in Carcinogenesis
}

\author{
Lokendra Kumar Sharma ${ }^{1, ~ *, ~ M e e n a k s h i ~ T i w a r i ~}{ }^{2}$, Santosh Kumar Mishra $^{1}$ \\ ${ }^{1}$ Biotechnology Program, Centre for Biological Sciences, Central University of Bihar, Patna, Bihar, India \\ ${ }^{2}$ Department of Laboratory Medicine and Pathology, All India Institute of Medical Sciences Patna, Bihar, India
}

Email address:

lokendra@cub.ac.in (L. K. Sharma)

\section{To cite this article:}

Lokendra Kumar Sharma, Meenakshi Tiwari, Santosh Kumar Mishra. Mitochondrial Alteration: A Major Player in Carcinogenesis. Cell Biology. Special Issue: Mitochondria: Implications in Human Health and Diseases. Vol. 3, No. 2-1, 2015, pp. 8-16.

doi: $10.11648 /$ j.cb.s. 2015030201.12

\begin{abstract}
In recent years we have witnessed mounting evidences on how a normal cell changes its cellular signaling and metabolic pathways to become highly proliferative cancer cell. Since mitochondrion is a major hub of energy production and several metabolic pathways, it is taking the center stage in defining alterations in energy homeostasis and metabolic rerouting of cancer cell proliferation. Similarly, mutations in the mitochondrial genome in cancer are providing new insights on how these mutations affect mitochondrial functions and change the oncogenic signaling and apoptosis mechanism. In this review, we will summarize these important mitochondrial mechanisms that contribute significantly in the progression of cancer. Further therapeutic approaches targeted to these altered mitochondrial mechanism in cancer are discussed. This review is a part of special issue on Mitochondria: implications in human health and diseases.
\end{abstract}

Keywords: Mitochondria, Metabolism, Reactive Oxygen Species and Cancer

\section{Introduction}

Mitochondria are central to cellular energy production and metabolism. Their role in regulating cellular signaling such as cell growth and proliferation and cell death pathways is expanding. As a consequence, mitochondrial dysfunctions have been implicated in a variety of clinical manifestations related to high energy requiring organs including heart, muscle, and brain [1]. Mitochondrial electron transport chain produces Reactive Oxygen Species (ROS), which are involved in the regulation of several physiological processes including cellular signaling, cell cycle, apoptosis and autophagy. Since these free radicals are highly active signaling molecules they can damage cellular components including mitochondrial DNA (mtDNA), proteins and lipids $[2,3]$. Specifically, if the damaged mtDNA is not repaired properly, it can incorporate mutations in mtDNA that could initiate tumorigenesis and sustain cancer development. To support the above notion, in recent years mtDNA mutations and dysfunctions have been reported in various cancers and provide us to further explore novel mitochondrial mechanisms in cancer progression [4]. Therefore how changes in mitochondrial metabolism and function affects the basic biology of cancer or determine the clinical outcome for cancer patients stands out as having unexplored diagnostic and therapeutic potential. In the following sections we will briefly discuss these different mitochondrial mechanisms and their potential usage for developing mitochondrial targeted therapeutic approaches.

\section{Alterations in Glycolytic Mechanism}

The significance of mitochondria in cancer came to highlight in early $20^{\text {th }}$ century, when Otto Warburg first proposed that origin of cancer was from non-neoplastic cells that adopted anaerobic metabolic conditions for their survival after defects in mitochondrial respiration [5]. It gave the idea that tumors were initiated by continuous damage to the mitochondria [6]. Subsequently, changes in the number, shape and function of mitochondria have been reported in various cancers [7]. However, several years later, it was found that mitochondrial defects are not common and most tumors retain normal oxidative phosphorylation and oxygen consumption capacity similar to normal tissues [8]. In fact, mitochondrial functions such as ROS generation are crucial for transformation in some systems [9]. Moreover some tumors, such as skin melanomas, lung carcinoma and breast cancer mostly rely on oxidative phosphorylation for fulfilling cellular demands in ATP [10]. Altered expression of phosphoglycerate dehydrogenase, phosphoglycerate mutase 1 , 
and pyruvate kinase $\mathrm{M} 2$ has been shown to reduce the rate of glycolytic flux to pyruvate and increase flux to biosynthetic pathways [11], such as serine biosynthesis [12] and the pentose phosphate pathway [13]. Several transcription factors (MYC and HIF1- $\alpha$ ), oncogenes (PI3K/AKT) and loss of tumor suppressor gene (P53) positively regulate glycolysis by up-regulating key glycolytic enzymes [14-16]. Therefore it is now broadly accepted that glycolytic mechanism evolved as an adaptation to hypoxic environment to fulfill ATP demand during the early avascular phase of tumor progression. Adaptation to the resulting acidic microenvironment that is caused by excess lactate production, may further drive the evolution of the glycolytic phenotype and allows effective shunting of carbon to key subsidiary biosynthetic pathways [17]. Several metabolic intermediates of TCA cycle are rerouted toward macromolecular synthesis that is critical to supplying enough nucleotides, proteins, and lipids for cell proliferation rather than providing ATP. Therefore, contrary to Warburg hypothesis, it is now becoming clearer that cancer cells do not inactivate mitochondrial energy metabolism, rather alter mitochondrial bioenergetics and biosynthesis rate.

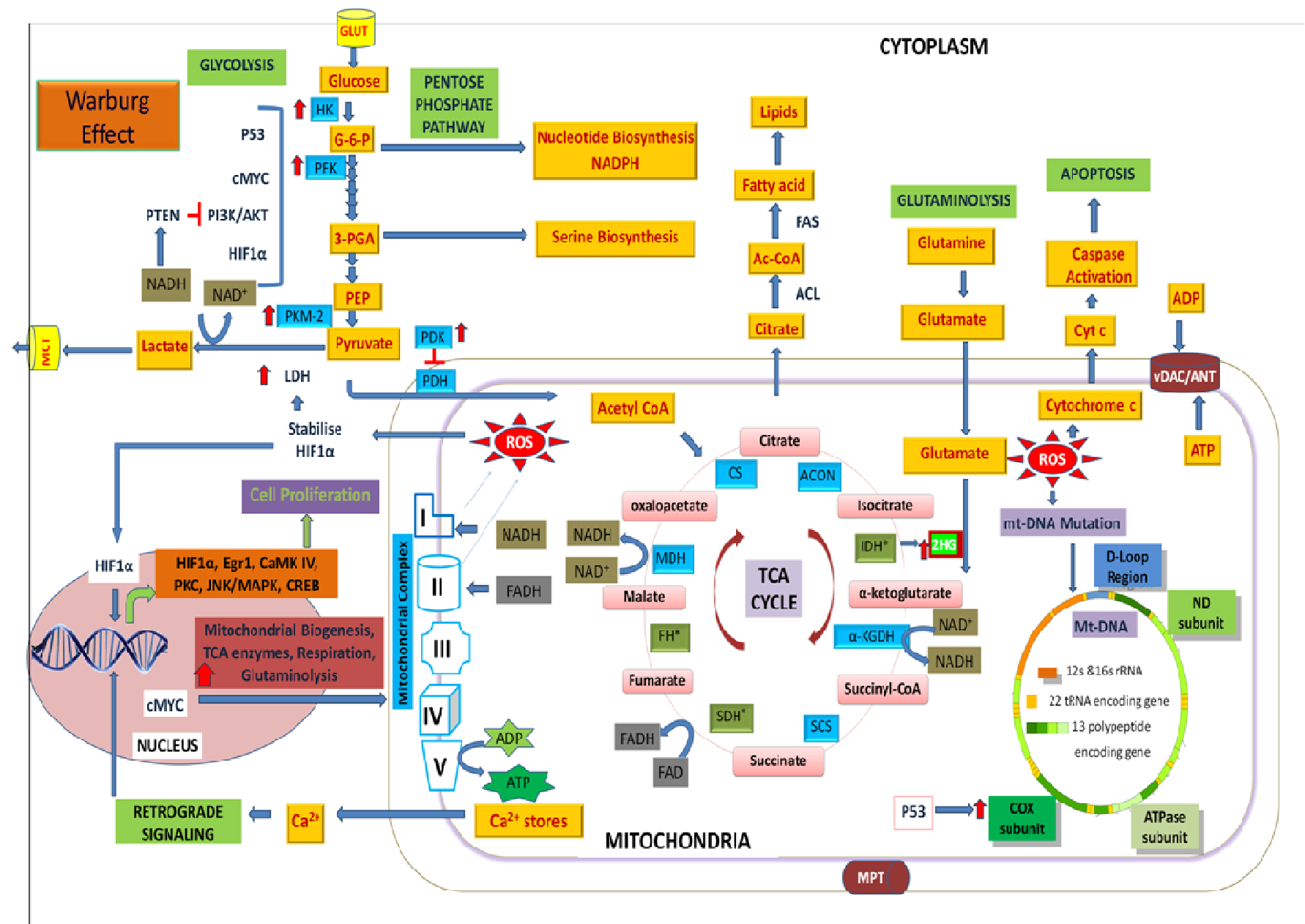

Figure 1. Schematic representation of metabolic alterations linked to mitochondria and cancer cell proliferation: The diagram shows some of the prominent aspects of metabolism in proliferating cell, including glycolysis; lactate production and changes in mitochondrial metabolic pathways. Resulting pyruvate from glycolysis is converted to lactate, which regenerates NAD+ from NADH and allows glycolysis to continue (Warburg). Lactate is secreted from the cell that provides acidic environment which support cell growth and proliferation. TCA cycle intermediates such as citrate serve as precursors for biosynthesis of lipids. TCA cycle replenishment (anaplerosis) is derived from the metabolism of glutamine to glutamate. Somatic mutation in TCA enzymes (as shown with *) such as IDH, SDH and FH may cause accumulation of intermediates or gain of a novel enzymatic activity. For example: IDH mutations causes the formation of oncometabolite 2-hydroxyglutarate $(2-H G)$ instead of $\alpha$-ketoglutarate $(\alpha K G)$. The PI3K, mTOR, p53, MYC and HIF1 participate in various steps of this metabolic phenotype by altering glycolysis and TCA cycle intermdiatelenzymes. Reactive oxygen species genetared through electron transfer may cause mutations in mitochondrial genome perticularily in different genes encoding subunits of respiratory complexes. (i.e. ND subunits for complex I, COX for complex $I V$, and D-loop region) and cause further ROS elevation, oncogenic activation or mitochondrial mediated apoptosis. Abbreviations: HK: Hexokinase, PFK: Phosphofructokinase, PKM-2: Pyruvate Kinase isoform M-2, PDK: Pyruvate dehydrogenase kinase, PDH: Pyruvate Dehydrogenase, LDH: Lactate dehydrogenase, MCT: Monocarboxylate transporters, G-6-P: Glucose-6-phosphate, 3-PGA: 3-Phosphoglyceric acid, NAD: Nicotinamide Adenine Dinucleotide (Oxidized), NADH: Nicotinamide adenine dinucleotide (Reduced), FAS: Fatty acid synthase, ACL: ATP citrate lyase, IDH: Isocitrate Dehydrogenase, SCS: Succinyl coenzyme A synthetase, SDH: Succinate Dhydrogenase, FH: Fumarate hydratase, MDH: Malate dehydrogenase, FADH: Flavin adenine dinucleotide (Reduced), HIF1:Hypoxia-inducible factor 1-alpha, EGr1: Early growth response protein 1, CaMKIV: Calcium/calmodulin dependent protein kinase type IV, PKC: Protein Kinase C, cMyc: Retroviral v- myconcogene. Upside red arrow shows up-regulation of respective enzyme/molecule/signaling. 


\section{Alterations in Mitochondrial Metabolic Pathways}

As discussed earlier, the link between mitochondria and cancer has historically focused on metabolism. In addition to the increase in the glycolytic metabolism, mitochondrial tricarboxylic acid (TCA) cycle has recently gained significant attention in the field of cancer metabolism. The end product of glycolysis pyruvate is converted to acetyl-CoA in the TCA cycle, is trans-aminated to alanine, or becomes lactate, particularly under hypoxic conditions. Citrate formation from acetyl-CoA and oxaloacetate allows TCA re-cycling, for generating electrons, $\mathrm{CO} 2$, and carbon skeletons for biosynthesis or anaplerosis. Citrate itself could be released into the cytosol and then changed to acetyl-CoA by an enzyme ATP citrate lyase (ACLY) for fatty acid synthesis and generation of bio-membranes. Major role of TCA cyle enzymes in cancer were first highlighted when mutations in nuclear genes of several key enzymes of TCA cycle including Isocitrate dehydrogenase (IDH), Succinate dehydrogenase (SDH) and Fumarate hydratase (FH) have identified in various tumors [18-20]. As a result alterations in their immediate or upstream metabolic products have been observed. For examples: oncogenic mutations confer a neomorphic activity to IDHs, which instead of converting isocitrate in 2-oxoglutarate (OG), reduce $\mathrm{OG}$ into 2-hydroxyglutarate $(\mathrm{R}-2 \mathrm{HG})$, which accumulates in cancer cells and shown to be an onco-metabolite [21]. Similarly, in FH deficient cells glutamine derived mitochondrial 2-oxoglutarate is metabolized in the reverse direction of the canonical TCA cycle to produce citrate by reductive carboxylation [22]. Not only the mutations in TCA cycle enzymes, cancer cells with respiratory complex III (cytochrome b-c1 complex) mutation also utilize similar glutamine-dependent reductive carboxylation rather than oxidative metabolism as the major pathway of citrate formation [23]. Glutamine addiction is one the other features of cancer cells where glutamine serve as a amino group donor for many biosynthetic pathways, including being the precursor for the synthesis of proline, ornithine, and arginine [24]. Similarly, glycine consumption and expression of the mitochondrial glycine biosynthetic pathway were strongly correlated with rates of proliferation across different cancer cell types [25]. In addition, fatty acid (FA) synthesis occurs at very high rates in tumor cells, which supports cancer growth by increasing availability of building blocks for cell membranes and for lipids containing molecules involved in cell signaling [26]. In conclusion, cells reprogram their metabolism to generate macromolecules or intermediates required for proliferation, whether it is by oxidative mitochondrial metabolism in normal cells or through alternative pathways.

\section{Mitochondrial DNA Mutation and Cancer}

The mammalian mitochondrial genome is a double stranded circular DNA of approximately $16 \mathrm{~kb}$ in size [27]. It contains 37 genes encoding 13 peptides for the respiratory chain complexes, 22 tRNAs and 2 rRNAs essential for protein synthesis within mitochondria. Besides these coding regions, a regulatory element known as displacement loop (D-loop) is also present which regulates mtDNA replication and transcription. The location of mtDNA is thought to be close to the electron transport chain or ROS generation site. Since mitochondria have relatively less sophisticated DNA protection or repair systems, mtDNA is more vulnerable to oxidative damage and results in somatic mutation. The mtDNA within a cell could be a blend of wild type and mutant, a condition called 'heteroplasmy'. One of the first comprehensive studies of mtDNA in cancer cells demonstrated that among 10 colorectal cancer lines, seven of them exhibited somatic mutations in their mtDNA encoded genes of respiratory complexes and rRNA genes [28]. Subsequently presence of different somatic mt-DNA mutations in wide array of human cancers including tumors of colon, breast, lung, prostate, liver, pancreas, kidney, thyroid, brain, gastric carcinoma and ovarian cancer, and nicely reviewed else ware $[4,29]$.

The significance of these mtDNA mutations on tumorigenesis has been tested with the cybrid (cytoplasmic hybrid) system, where mtDNA is singled out for analysis [30]. Cybrids carrying pathogenic mutations i.e. in ATP synthase subunit 6 gene (ATP6), conferred an advantage in the early stage of tumor growth [31,32]. The contribution of these mtDNA mutations at early stage is critically important as in the beginning phase, cancer cell encounter more oxidative insult and start accumulating mutation in mitochondrial DNA (heteroplasmy). It was confirmed in a unique study where heteroplasmic mutations in complex I subunit gene was shown to contribute significantly in increasing tumorigenic potential than homoplasmic mutation [33]. When mitochondrial functions were analyzed in heteroplasmic vs homoplasmic mtDNA mutant conditions, homoplasmic appeared to be severely impaired. However heteroplasmic mutation contributed in increased tumorigenic potential that is mediated through elevated mitochondrial ROS mediated AKT activation and apoptosis resistance [34]. Not only the mtDNA mutations enhance the tumorigenicity, such mutations are also capable of enhancing metastatic potential of poorly metastatic cells and can metastasize to other organs such as bone by increasing growth factors and affecting tumor microenvironment $[35,36]$.

The major link between mtDNA mutations to tumorigenesis is the alteration of ROS level, however it is highly controversial as ROS are involved in various cellular signaling in normal cells as well. Since mtDNA less cells (Rho-Zero) 
cells do not generate elevated ROS they do not grow in an anchor-independent way as cancer cells [9]. Similarly, heteroplasmic mutations in ND5 mitochondrial gene (complex I) show higher ROS production and tumor growth than the same mutations in a homoplasmic state [33]. Further overproduction of ROS was observed in cells with mutation in ND6 subunit of complex I and contributed in enhancing metastasis potential $[35,37]$. Overall these studies support the idea of ROS involvement in tumorigenesis and metastasis. However, in some cases no enhanced ROS and high metastatic potential was also observed for the cells with large mtDNA deletion in cybrid system [38]. This effect could be explain for the fact that as the respiratory chain is a ROS producer, when it is completely inhibited, ROS levels are not elevated and do not influence tumor formation and metastasis. It is also worth mentioning that partial inhibition not complete inhibition of respiratory chain supports the enhanced tumorigenic potential as discussed for heteroplasmic mtDNA mutation induced oncogenic activation and tumorigenesis earlier [33, 34].

\section{Mitochondrial Signaling Mechanisms}

Mitochondria exhibit both vital and harmful functions in physiological and pathological conditions respectively. Mitochondria contribute in cellular signaling via three core functions; those are ATP production through oxidative phosphorylation, generation of ROS which serve as important signaling molecules, and apoptosis regulation. Since cancer cells are highly proliferative, they consume more oxygen to up-regulate TCA cycle for ATP requirement and thus, tumor tissues suffer from hypoxia. The transcription factor, HIF-1(Hypoxia inducible factor-1), is one of the key mediators of the hypoxia response through regulating genes involved in metabolism, angiogenesis, cell cycle and apoptosis [39]. In addition to up-regulating the glycolytic pathway, HIF-1 was also shown to inhibit mitochondrial biogenesis and respiration in a renal cell carcinoma model by repression of C-MYC activity [40]. Importantly, C-MYC is required for the expression of co-activator PGC-1, which is a key regulator of mitochondrial biogenesis [41]. Alternatively, HIF-1 down regulates oxidative phosphorylation through regulating pyruvate dehydrogenase, which converts pyruvate to acetyl-CoA $[42,43]$. Interestingly, it was also demonstrated that HIF-1 regulates complex IV subunits and alters respiration efficiency in hypoxic cells [44]. Such regulation has important implications in ATP production, oxygen consumption and ROS generation. HIF1 is shown to be activated by alterations in ROS levels due to mtDNA mutations and regulates tumorigenesis and metastasis [34, 35] Studies have indicated that mitochondrial respiration defects led to elevated levels of redox molecules such as NADH, which could subsequently inactivate PTEN [45]. Inhibition of PTEN could activate protein kinase B (Akt), and Akt was shown to enhance glycolysis, possibly through the effects on its key rate-limiting step, phosphorylation of newly acquired glucose by hexokinases [46]. Akt alone is sufficient to up regulate glycolysis and also triggers an increase in cell survival which is commonly observed in cancer cells [16]. The tumor suppressor p53 favors ATP production by OXPHOS and decreases the cellular ROS production by inducing TP53 Induced Glycolysis and Apoptosis Regulator (TIGAR) [47]. p53 also up-regulates OXPHOS complex IV by the induction of the Cytochrome c Oxidase (COX) $\mathrm{Cu} 2+$ chaperone, $\mathrm{SCO} 2$ [48].

Defects in the respiratory chain could either promote or inhibit cell death, depending on the specific alteration in electron flow [49]. It is primarily regulated by ROS level; however the role of ROS in cancer is still highly debatable. On one hand defective mitochondrial functions may up-regulate ROS levels which in turn can activate oncogenic signaling as discussed earlier. On the other hand these free radicals can easily diffuse to cytosol it can damage various cellular components such as lipid, protein and DNA [50]. Therefore, excessive ROS levels may also induce apoptosis through activation of death receptor, Caspases dependent and independent pathways [51]. Recently altered levels of redox molecules such as NAD and NADH have shown to regulate tumor metastasis through mTOR and autophagy [52].

The communication pathway from the mitochondria to the nucleus is known as retrograde signaling that is used to describe the cellular response to the changes in the functions of mitochondria as mentioned above. One of the mechanisms suggested to play a role in the retrograde response is mitochondrial stress, which is supported by changes in mitochondrial membrane potential and elevation of calcium levels [53]. This increased calcium level results in the activation of calcium responsive genes/proteins such as CaMKIV (calcium/ calmodulin kinase IV) and CREB (cAMP-responsive element-binding protein) and Egr1 (early growth response gene-1) through PKC-mediated phosphorylation $[54,55]$. Retrograde signaling induces the expression of a number of tumor-specific marker genes, such as extracellular matrix protease, TGF- $\beta$ and epiregulin, as well as other genes that control cell growth and proliferation, such as PKC, JNK/MAPK, CREB and NF-kB [53]. On the other side, mitochondrial calcium accumulation also causes cell death which is implicated in neuro-degenerative diseases [56].

Overall, molecular damages elicited by altered signaling molecules through mitochondria in cancer determine the fate of cells to either repair the damage or, lead to activate the cell death program.

\section{Mitochondrial Therapeutics}

\subsection{Metabolic Targets}

Mitochondria are attractive targets in developing approaches for cancer therapy due to their involvement in variety of functions. Considering Warburg effect as a major driving force of glucose utilization and ATP production for proliferating cells, inhibition of glycolysis has been found useful for preventing cancer cell proliferation [57]. Various enzymatic inhibitors of glycolysis inhibitors of HK such as 
2-deoxyglucose (2-DG), lonidamine (LON, 1[(2,4-dichlorophenyl)methyl] -1H-indazole- 3-carboxylic acid) and 3-bromopyruvate (3-BrPA) are currently in pre-clinical and early phase clinical trials. Hexokinase is frequently overexpressed in human tumours and $\mathrm{HK}$ binds to vDAC more tightly in cancer cells than in normal cells. Disrupting the interactions of $\mathrm{HK}$ and $\mathrm{vDAC}$ have shown preferential killing of tumor cells and therefore it offers another intriguing target to selectively trigger cancer cell death [58]. Similarly pharmacological and genetic inhibition of other metabolic enzymes such as pyruvate dehydrogenase by dicholoroacetate, Lactate dehydrogenase (LDGH-A) by shRNA and ATP citrate Lyase (ACL) by SB-204990 have shown promising results on cancer regression in different models [59-61]. Since glutamine addiction is one of the features of cancer cells, modulation of glutaminase (glutamine to glutamate) and glutamate dehydrogenase (glutamate to $\alpha-\mathrm{KG}$ ) activities that affect glutamine metabolism, have been proposed to inhibit tumor growth [62]. $\mathrm{MYC}$ is also one of the important transcription factors which up regulates several key enzymes involved glycolysis and TCA cycle, and increases mitochondrial OXPHOS and biogenesis $[63,64]$. It could be a potential therapeutic target in cancer therapy and therefore several small molecule inhibitors are known to inhibit MYC expression and shown to be effective against tumor growth [65].

\subsection{Targeting Reactive Oxygen Species}

Since cancer cells experience deregulated ROS levels, it serves as a dual edge sword. ROS level may activate oncogenic signaling however beyond threshold it may cause cell death. Targeting ROS level above the threshold has been central in various cancer therapies and therefore, it has been exploited experimentally in many studies to target cancer cells [66]. In fact, some current genotoxic agents used in the clinic, such as Cisplatin and certain alkaloids rely on ROS production for their efficacy [67]. However, existing anti-cancer therapies shows deleterious side effects on normal tissues, partially triggered by ROS, which limit the applicability and their anti-tumor activity. Alternatively, the antioxidant therapy has shown promising results in laboratory animals however in clinical trials it gave mixed results (http:// www.cancer.gov/ cancertopics/ factsheet/ prevention/ antioxidants). Natural compounds have gain significant attention in recent years due to their low side effects and cost effectiveness. Majority of these compounds target mitochondrial metabolism and induces alteration in ROS and mitochondrial mediated apoptosis [68]. Further studies indicated their role in inducing apoptosis via inhibition of mitochondrial respiration and increased ROS production [69]. Ongoing researches are focused on overcoming these side-effects, combining with radiotherapy or chemotherapy without altering the efficacy of the therapy. Conversely, several intracellular molecules and enzymes such as glutathione, glutathione peroxidase, glutathione transferase, catalase, superoxide dismutase, and a variety of other antioxidants regulates the ROS levels that allows cells to maintain homeostasis and to prevent oxidative stress [70]. Therefore use of antioxidants which targets specifically normal or cancer cell mitochondria such as (SS-31 and Mito-Q) may be useful to reduce oxidative stress in normal cell before their transformation to cancer cells.

\subsection{Targating Mitochondrial Functions}

A proper mitochondrial membrane potential is required for exchange of small molecules through mitochondrial trans-membrane pore complex (PTPC), ATP generation and for Calcium regulation. However when it is deregulated it causes membrane permeability transition (MPT) and subsequently cytosolic calcium overload, release of apoptotic protein and apoptosis induction. Therefore targeting PTPC in particular Adenine nucleotide translocase (ANT) may cause inhibition of ATP/ADP transport, ATP depletion, ROS overproduction, mitochondrial depolarization and induction of mitochondrial mediated apoptosis pathway. These ANT ligands (i.e. 4- (N- (S-glutathionylacetyl) amino) phenylarsenoxide (GSAO) and lonidamine) could be useful for mediating apoptosis through mitochondria [71, 72]. As discussed in previous sections that specific mtDNA mutations in respiratory chain complexes cause mitochondrial dysfunctions and enhance tumorigenesis. These mitochondrial dysfunctions could be corrected using genetic approaches such as introducing alternative enzymes complementing defective respiratory enzymes. For example, Yeast NDI1 has shown promising results in restoring mitochondrial functions in CI defective systems [73]. In particular this Yeast NDI1 has shown significant inhibition in tumorigenesis in mitochondrial models of cancer [34, 52]. Overall, balance of mitochondrial functions could be exploited to develop therapeutic approaches targeting cancer cell mitochondria.

\subsection{Targeting Autophagy}

Autophagy is a cellular degradation pathway for the clearance and recycling of damaged organelles including mitochondria (mitophagy). Selective elimination of damaged mitochondria by mitophagy was found to be an important mitochondrial quality control mechanism [74]. It is also one of the cell death mechanisms other than apoptosis. The role of autophagy in cancer is complex and it can act to either promote or inhibit tumorigenesis depending on tumor type, stage, and genetic context (Ref). Knockdown of essential autophagy genes in tumor cells has been shown to induce cell death suggesting its role in tumor cell survival [75]. ROS can trigger mitochondrial dysfunctions and subsequently autophagy/mitophagy to provide high energy metabolites for tumor microenvironment that helps in cancer cell proliferation [76]. Oncogene such as RAS has shown to activate mitophagy for cancer cell survival by enhancing glycolysis [77]. Therefore, targeting autophagy / mitophagy in such cases may provide a potential therapeutic approach. Inhibition of autophagy in certain cancer cell lines (i.e. pancreatic) led to tumor regression and extended survival in pancreatic cancer 
xenografts and genetic mouse models [78]. It is perticulariy important in cases where cancer cell survive after chemotherapy and/or radiation, and activation of autophagy may enable a state of dormancy in residual cancer cells that may contribute to tumor recurrence and progression later. Some of the autophagy inhibitors (i.e. cholorquinone and hydrochloroquinone) in combination with radiation or other chemotherapies are under clinical trials for various cancers [79]. Since autophagy can act as a cell death mechanism, it is also worth mentioning that in some cases defect in autophagic pathway increases tumorigenesis. For example during autophagy inhibition, one of the markers of autophaguc flux p62/SQSTM 1 protein aggregates, causes mitochondrial damge, and misfolded proteins lead to the production of ROS and genomic instability [80]. In such cases induction of autophagy can serve as an anti-cancer mechanism. For a number of drugs, combining ROS and autophagy inducers could facilitate tumor killing activity [81]. Overall, most of the preclinical evidence supports autophagy as a novel therapeutic target whose modulation presents new opportunities for cancer treatment.

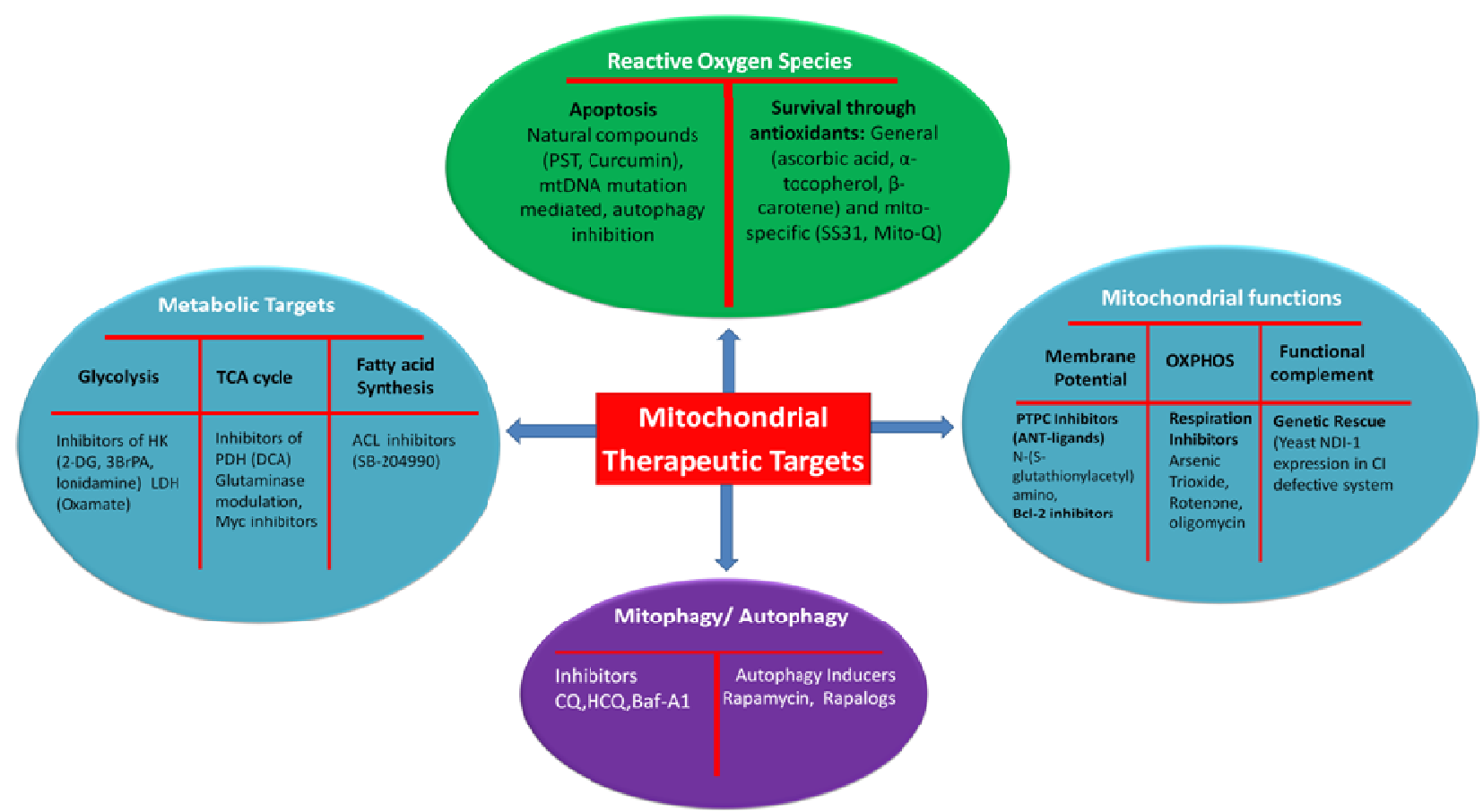

Figure 2. Potential of mitochondria as a target for anti-cancer therapy. Different factors linked to mitochondria may be used as direct or indirect target for cancer cell mitochondria. Metabolic targets involve inhibitors of various metabolic pathways enzymes. ROS activation may be useful in inducing mitochondrial mediated cell death in cancer cells and conversely antioxidant treatment in normal cells may limit the ROS level below threshold required for oncogene activation. Similarily targeting mitochondrial functions such as altering the membrane permeability or inhibiting respiration may lead to mitochondrial mediated apoptosis. Functional complementation of defective RC complexes through genetic approaches (i.e. single subunit enzyme of Yeast NDI1 for complex I) may provide alternative approach to rescue mitochondrial functions in cancer cells. Since autophagy functions both ways, therefore accordingly it's inducers or inhibitors could be utilized to develop therapeutic approaches. Abbreviations: 2DG: 2-Deoxy-D-glucose, 3BrPA: 3-bromo-2-oxopropanoic acid, DCA: Dichloroacetate, CQ: chloroquine, HCQ: hydroxychloroquine, Baf-A1: Bafilomycin A1, SS-31: d-Arg-2', 6'-dimethyltyrosine-Lys-Phe-NH , PST: Pancratistatin,

\section{Perspective}

In summary, ongoing researches on mitochondrial mechanisms and metabolism in cancer have provided immense potential for the development of targeted therapeutics. However, many mysteries remain unsolved in our understanding of the role of mitochondria in normal vs. cancer cells. Analysis of the physiological consequences of these mitochondrial alterations may further provide new insight into how changes in mitochondrial metabolism can alter cell fate.

\section{Acknowledgments}

The authors laboratory is supported by Science and Engineering Research Board (SERB), Government of India's
Scheme for Young Scientist research grant to Lokendra Kumar Sharma (SB/YS/LS-95/2013). Authors would like to thank Dr. Yidong Bai (The University of Texas Health Science Center at San Antonio) for his valuable suggestions to improve the manuscript.

\section{References}

[1] D. C. Wallace, A mitochondrial paradigm of metabolic and degenerative diseases, aging, and cancer: a dawn for evolutionary medicine, Annual review of genetics, 39 (2005) 359-407.

[2] B. N. Ames, M. K. Shigenaga, Oxidants are a major contributor to aging, Annals of the New York Academy of Sciences, 663 (1992) 85-96. 
[3] D. Harman, Role of free radicals in aging and disease, Annals of the New York Academy of Sciences, 673 (1992) 126-141.

[4] A. Chatterjee, E. Mambo, D. Sidransky, Mitochondrial DNA mutations in human cancer, Oncogene, 25 (2006) 4663-4674.

[5] O. Warburg, F. Wind, E. Negelein, The Metabolism of Tumors in the Body, The Journal of general physiology, 8 (1927) 519-530.

[6] O. Warburg, On respiratory impairment in cancer cells, Science, 124 (1956) 269-270.

[7] P.L. Pedersen, Tumor mitochondria and the bioenergetics of cancer cells, Progress in experimental tumor research, 22 (1978) 190-274.

[8] S. Weinhouse, The Warburg hypothesis fifty years later, Zeitschrift fur Krebsforschung und klinische Onkologie. Cancer research and clinical oncology, 87 (1976) 115-126.

[9] F. Weinberg, R. Hamanaka, W.W. Wheaton, S. Weinberg, J. Joseph, M. Lopez, B. Kalyanaraman, G.M. Mutlu, G.R. Budinger, N.S. Chandel, Mitochondrial metabolism and ROS generation are essential for Kras-mediated tumorigenicity, Proceedings of the National Academy of Sciences of the United States of America, 107 (2010) 8788-8793.

[10] R. Moreno-Sanchez, S. Rodriguez-Enriquez, A. Marin-Hernandez, E. Saavedra, Energy metabolism in tumor cells, The FEBS journal, 274 (2007) 1393-1418.

[11] J.W. Locasale, A.R. Grassian, T. Melman, C.A. Lyssiotis, K.R. Mattaini, A.J. Bass, G. Heffron, C.M. Metallo, T. Muranen, H. Sharfi, A.T. Sasaki, D. Anastasiou, E. Mullarky, N.I. Vokes, M. Sasaki, R. Beroukhim, G. Stephanopoulos, A.H. Ligon, M. Meyerson, A.L. Richardson, L. Chin, G. Wagner, J.M. Asara, J.S. Brugge, L.C. Cantley, M.G. Vander Heiden, Phosphoglycerate dehydrogenase diverts glycolytic flux and contributes to oncogenesis, Nature genetics, 43 (2011) 869-874.

[12] R. Possemato, K.M. Marks, Y.D. Shaul, M.E. Pacold, D. Kim, K. Birsoy, S. Sethumadhavan, H.K. Woo, H.G. Jang, A.K. Jha, W.W. Chen, F.G. Barrett, N. Stransky, Z.Y. Tsun, G.S. Cowley, J. Barretina, N.Y. Kalaany, P.P. Hsu, K. Ottina, A.M. Chan, B. Yuan, L.A. Garraway, D.E. Root, M. Mino-Kenudson, E.F. Brachtel, E.M. Driggers, D.M. Sabatini, Functional genomics reveal that the serine synthesis pathway is essential in breast cancer, Nature, 476 (2011) 346-350.

[13] T. Hitosugi, L. Zhou, S. Elf, J. Fan, H.B. Kang, J.H. Seo, C. Shan, Q. Dai, L. Zhang, J. Xie, T.L. Gu, P. Jin, M. Aleckovic, G. LeRoy, Y. Kang, J.A. Sudderth, R.J. DeBerardinis, C.H. Luan, G.Z. Chen, S. Muller, D.M. Shin, T.K. Owonikoko, S. Lonial, M.L. Arellano, H.J. Khoury, F.R. Khuri, B.H. Lee, K. Ye, T.J. Boggon, S. Kang, C. He, J. Chen, Phosphoglycerate mutase 1 coordinates glycolysis and biosynthesis to promote tumor growth, Cancer cell, 22 (2012) 585-600.

[14] G. L. Semenza, HIF-1: upstream and downstream of cancer metabolism, Current opinion in genetics \& development, 20 (2010) 51-56.

[15] G. Qing, N. Skuli, P.A. Mayes, B. Pawel, D. Martinez, J.M. Maris, M.C. Simon, Combinatorial regulation of neuroblastoma tumor progression by $\mathrm{N}-\mathrm{Myc}$ and hypoxia inducible factor HIF-1alpha, Cancer research, 70 (2010) 10351-10361.
[16] R.L. Elstrom, D.E. Bauer, M. Buzzai, R. Karnauskas, M.H. Harris, D.R. Plas, H. Zhuang, R.M. Cinalli, A. Alavi, C.M. Rudin, C.B. Thompson, Akt stimulates aerobic glycolysis in cancer cells, Cancer research, 64 (2004) 3892-3899.

[17] S.Y. Lunt, M.G. Vander Heiden, Aerobic glycolysis: meeting the metabolic requirements of cell proliferation, Annual review of cell and developmental biology, 27 (2011) 441-464.

[18] B.E. Baysal, R.E. Ferrell, J.E. Willett-Brozick, E.C. Lawrence, D. Myssiorek, A. Bosch, A. van der Mey, P.E. Taschner, W.S. Rubinstein, E.N. Myers, C.W. Richard, 3rd, C.J. Cornelisse, P. Devilee, B. Devlin, Mutations in SDHD, a mitochondrial complex II gene, in hereditary paraganglioma, Science, 287 (2000) 848-851.

[19] I. P. Tomlinson, N. A. Alam, A. J. Rowan, E. Barclay, E. E. Jaeger, D. Kelsell, I. Leigh, P. Gorman, H. Lamlum, S. Rahman, R. R. Roylance, S. Olpin, S. Bevan, K. Barker, N. Hearle, R. S. Houlston, M. Kiuru, R. Lehtonen, A. Karhu, S. Vilkki, P. Laiho, C. Eklund, O. Vierimaa, K. Aittomaki, M. Hietala, P. Sistonen, A. Paetau, R. Salovaara, R. Herva, V. Launonen, L. A. Aaltonen, C. Multiple Leiomyoma, Germline mutations in FH predispose to dominantly inherited uterine fibroids, skin leiomyomata and papillary renal cell cancer, Nature genetics, 30 (2002) 406-410.

[20] H. Yan, D. W. Parsons, G. Jin, R. McLendon, B. A. Rasheed, W. Yuan, I. Kos, I. Batinic-Haberle, S. Jones, G. J. Riggins, H. Friedman, A. Friedman, D. Reardon, J. Herndon, K. W. Kinzler, V. E. Velculescu, B. Vogelstein, D. D. Bigner, IDH1 and IDH2 mutations in gliomas, The New England journal of medicine, 360 (2009) 765-773.

[21] L. Dang, D. W. White, S. Gross, B. D. Bennett, M. A. Bittinger, E. M. Driggers, V. R. Fantin, H. G. Jang, S. Jin, M. C. Keenan, K. M. Marks, R. M. Prins, P. S. Ward, K. E. Yen, L. M. Liau, J. D. Rabinowitz, L. C. Cantley, C. B. Thompson, M. G. Vander Heiden, S. M. Su, Cancer-associated IDH1 mutations produce 2-hydroxyglutarate, Nature, 462 (2009) 739-744.

[22] L. B. Sullivan, N. S. Chandel, Mitochondrial metabolism in TCA cycle mutant cancer cells, Cell cycle, 13 (2014) 347-348.

[23] A. R. Mullen, W. W. Wheaton, E. S. Jin, P. H. Chen, L. B. Sullivan, T. Cheng, Y. Yang, W. M. Linehan, N. S. Chandel, R. J. DeBerardinis, Reductive carboxylation supports growth in tumour cells with defective mitochondria, Nature, 481 (2012) 385-388.

[24] C. T. Hensley, A. T. Wasti, R. J. DeBerardinis, Glutamine and cancer: cell biology, physiology, and clinical opportunities, The Journal of clinical investigation, 123 (2013) 3678-3684.

[25] M. Jain, R. Nilsson, S. Sharma, N. Madhusudhan, T. Kitami, A.L. Souza, R. Kafri, M.W. Kirschner, C.B. Clish, V.K. Mootha, Metabolite profiling identifies a key role for glycine in rapid cancer cell proliferation, Science, 336 (2012) 1040-1044.

[26] E. Currie, A. Schulze, R. Zechner, T.C. Walther, R.V. Farese, Jr., Cellular fatty acid metabolism and cancer, Cell metabolism, 18 (2013) 153-161.

[27] S. Anderson, A.T. Bankier, B.G. Barrell, M.H. de Bruijn, A.R. Coulson, J. Drouin, I.C. Eperon, D.P. Nierlich, B.A. Roe, F. Sanger, P.H. Schreier, A.J. Smith, R. Staden, I.G. Young, Sequence and organization of the human mitochondrial genome, Nature, 290 (1981) 457-465. 
[28] K. Polyak, Y. Li, H. Zhu, C. Lengauer, J.K. Willson, S.D. Markowitz, M.A. Trush, K.W. Kinzler, B. Vogelstein, Somatic mutations of the mitochondrial genome in human colorectal tumours, Nature genetics, 20 (1998) 291-293.

[29] J. Lu, L.K. Sharma, Y. Bai, Implications of mitochondrial DNA mutations and mitochondrial dysfunction in tumorigenesis, Cell research, 19 (2009) 802-815.

[30] M. P. King, G. Attardi, Human cells lacking mtDNA: repopulation with exogenous mitochondria by complementation, Science, 246 (1989) 500-503.

[31] Y. Shidara, K. Yamagata, T. Kanamori, K. Nakano, J.Q. Kwong, G. Manfredi, H. Oda, S. Ohta, Positive contribution of pathogenic mutations in the mitochondrial genome to the promotion of cancer by prevention from apoptosis, Cancer research, 65 (2005) 1655-1663.

[32] J. A. Petros, A.K. Baumann, E. Ruiz-Pesini, M.B. Amin, C.Q. Sun, J. Hall, S. Lim, M.M. Issa, W.D. Flanders, S.H. Hosseini, F.F. Marshall, D.C. Wallace, mtDNA mutations increase tumorigenicity in prostate cancer, Proceedings of the National Academy of Sciences of the United States of America, 102 (2005) 719-724.

[33] J.S. Park, L.K. Sharma, H. Li, R. Xiang, D. Holstein, J. Wu, J. Lechleiter, S.L. Naylor, J.J. Deng, J. Lu, Y. Bai, A heteroplasmic, not homoplasmic, mitochondrial DNA mutation promotes tumorigenesis via alteration in reactive oxygen species generation and apoptosis, Human molecular genetics, 18 (2009) 1578-1589.

[34] L.K. Sharma, H. Fang, J. Liu, R. Vartak, J. Deng, Y. Bai, Mitochondrial respiratory complex I dysfunction promotes tumorigenesis through ROS alteration and AKT activation, Human molecular genetics, 20 (2011) 4605-4616.

[35] K. Ishikawa, K. Takenaga, M. Akimoto, N. Koshikawa, A. Yamaguchi, H. Imanishi, K. Nakada, Y. Honma, J. Hayashi, ROS-generating mitochondrial DNA mutations can regulate tumor cell metastasis, Science, 320 (2008) 661-664.

[36] R.S. Arnold, C.Q. Sun, J.C. Richards, G. Grigoriev, I.M. Coleman, P.S. Nelson, C.L. Hsieh, J.K. Lee, Z. Xu, A. Rogatko, A.O. Osunkoya, M. Zayzafoon, L. Chung, J.A. Petros, Mitochondrial DNA mutation stimulates prostate cancer growth in bone stromal environment, The Prostate, 69 (2009) 1-11.

[37] K. Ishikawa, N. Koshikawa, K. Takenaga, K. Nakada, J. Hayashi, Reversible regulation of metastasis by ROS-generating mtDNA mutations, Mitochondrion, 8 (2008) 339-344.

[38] M. Kulawiec, K.M. Owens, K.K. Singh, Cancer cell mitochondria confer apoptosis resistance and promote metastasis, Cancer biology \& therapy, 8 (2009) 1378-1385.

[39] G.L. Semenza, Hypoxia-inducible factors in physiology and medicine, Cell, 148 (2012) 399-408.

[40] H. Zhang, P. Gao, R. Fukuda, G. Kumar, B. Krishnamachary, K.I. Zeller, C.V. Dang, G.L. Semenza, HIF-1 inhibits mitochondrial biogenesis and cellular respiration in VHL-deficient renal cell carcinoma by repression of C-MYC activity, Cancer cell, 11 (2007) 407-420.

[41] F. Li, Y. Wang, K.I. Zeller, J.J. Potter, D.R. Wonsey, K.A. O'Donnell, J.W. Kim, J.T. Yustein, L.A. Lee, C.V. Dang, Myc stimulates nuclearly encoded mitochondrial genes and mitochondrial biogenesis, Molecular and cellular biology, 25 (2005) 6225-6234.
[42] J.W. Kim, I. Tchernyshyov, G.L. Semenza, C.V. Dang, HIF-1-mediated expression of pyruvate dehydrogenase kinase: a metabolic switch required for cellular adaptation to hypoxia, Cell metabolism, 3 (2006) 177-185.

[43] I. Papandreou, R.A. Cairns, L. Fontana, A.L. Lim, N.C. Denko, HIF-1 mediates adaptation to hypoxia by actively downregulating mitochondrial oxygen consumption, Cell metabolism, 3 (2006) 187-197.

[44] R. Fukuda, H. Zhang, J.W. Kim, L. Shimoda, C.V. Dang, G.L. Semenza, HIF-1 regulates cytochrome oxidase subunits to optimize efficiency of respiration in hypoxic cells, Cell, 129 (2007) 111-122.

[45] H. Pelicano, R.H. Xu, M. Du, L. Feng, R. Sasaki, J.S. Carew, Y. Hu, L. Ramdas, L. Hu, M.J. Keating, W. Zhang, W. Plunkett, P. Huang, Mitochondrial respiration defects in cancer cells cause activation of Akt survival pathway through a redox-mediated mechanism, The Journal of cell biology, 175 (2006) 913-923.

[46] R.B. Robey, N. Hay, Mitochondrial hexokinases, novel mediators of the antiapoptotic effects of growth factors and Akt, Oncogene, 25 (2006) 4683-4696.

[47] K. Bensaad, A. Tsuruta, M.A. Selak, M.N. Vidal, K. Nakano, R Bartrons, E. Gottlieb, K.H. Vousden, TIGAR, a p53-inducible regulator of glycolysis and apoptosis, Cell, 126 (2006) 107-120.

[48] S. Matoba, J.G. Kang, W.D. Patino, A. Wragg, M. Boehm, O. Gavrilova, P.J. Hurley, F. Bunz, P.M. Hwang, p53 regulates mitochondrial respiration, Science, 312 (2006) 1650-1653.

[49] J.Q. Kwong, M.S. Henning, A.A. Starkov, G. Manfredi, The mitochondrial respiratory chain is a modulator of apoptosis, The Journal of cell biology, 179 (2007) 1163-1177.

[50] M. Ott, V. Gogvadze, S. Orrenius, B. Zhivotovsky, Mitochondria, oxidative stress and cell death, Apoptosis : an international journal on programmed cell death, 12 (2007) 913-922.

[51] I.R. Indran, G. Tufo, S. Pervaiz, C. Brenner, Recent advances in apoptosis, mitochondria and drug resistance in cancer cells, Biochimica et biophysica acta, 1807 (2011) 735-745.

[52] A.F. Santidrian, A. Matsuno-Yagi, M. Ritland, B.B. Seo, S.E. LeBoeuf, L.J. Gay, T. Yagi, B. Felding-Habermann, Mitochondrial complex I activity and NAD+/NADH balance regulate breast cancer progression, The Journal of clinical investigation, 123 (2013) 1068-1081.

[53] R.A. Butow, N.G. Avadhani, Mitochondrial signaling: the retrograde response, Molecular cell, 14 (2004) 1-15.

[54] T. Arnould, S. Vankoningsloo, P. Renard, A. Houbion, N. Ninane, C. Demazy, J. Remacle, M. Raes, CREB activation induced by mitochondrial dysfunction is a new signaling pathway that impairs cell proliferation, The EMBO journal, 21 (2002) 53-63.

[55] D. Freyssenet, I. Irrcher, M.K. Connor, M. Di Carlo, D.A. Hood, Calcium-regulated changes in mitochondrial phenotype in skeletal muscle cells, American journal of physiology. Cell physiology, 286 (2004) C1053-1061.

[56] F. Celsi, P. Pizzo, M. Brini, S. Leo, C. Fotino, P. Pinton, R. Rizzuto, Mitochondria, calcium and cell death: a deadly triad in neurodegeneration, Biochimica et biophysica acta, 1787 (2009) 335-344. 
[57] H. Pelicano, D.S. Martin, R.H. Xu, P. Huang, Glycolysis inhibition for anticancer treatment, Oncogene, 25 (2006) 4633-4646.

[58] J.G. Pastorino, J.B. Hoek, N. Shulga, Activation of glycogen synthase kinase 3 beta disrupts the binding of hexokinase II to mitochondria by phosphorylating voltage-dependent anion channel and potentiates chemotherapy-induced cytotoxicity, Cancer research, 65 (2005) 10545-10554.

[59] S. Bonnet, S.L. Archer, J. Allalunis-Turner, A. Haromy, C. Beaulieu, R. Thompson, C.T. Lee, G.D. Lopaschuk, L. Puttagunta, S. Bonnet, G. Harry, K. Hashimoto, C.J. Porter, M.A. Andrade, B. Thebaud, E.D. Michelakis, A mitochondria-K+ channel axis is suppressed in cancer and its normalization promotes apoptosis and inhibits cancer growth, Cancer cell, 11 (2007) 37-51.

[60] V.R. Fantin, J. St-Pierre, P. Leder, Attenuation of LDH-A expression uncovers a link between glycolysis, mitochondrial physiology, and tumor maintenance, Cancer cell, 9 (2006) 425-434.

[61] G. Hatzivassiliou, F. Zhao, D.E. Bauer, C. Andreadis, A.N. Shaw, D. Dhanak, S.R. Hingorani, D.A. Tuveson, C.B. Thompson, ATP citrate lyase inhibition can suppress tumor cell growth, Cancer cell, 8 (2005) 311-321.

[62] J. B. Wang, J. W. Erickson, R. Fuji, S. Ramachandran, P. Gao, R. Dinavahi, K.F. Wilson, A.L. Ambrosio, S.M. Dias, C.V. Dang, R.A. Cerione, Targeting mitochondrial glutaminase activity inhibits oncogenic transformation, Cancer cell, 18 (2010) 207-219.

[63] C. V. Dang, MYC, metabolism, cell growth, and tumorigenesis, Cold Spring Harbor perspectives in medicine, 3 (2013).

[64] J. A. Graves, Y. Wang, S. Sims-Lucas, E. Cherok, K. Rothermund, M.F. Branca, J. Elster, D. Beer-Stolz, B. Van Houten, J. Vockley, E.V. Prochownik, Mitochondrial structure, function and dynamics are temporally controlled by c-Myc, PloS one, 7 (2012) e37699.

[65] S. Fletcher, E.V. Prochownik, Small-molecule inhibitors of the Myc oncoprotein, Biochimica et biophysica acta, (2014).

[66] L. Raj, T. Ide, A.U. Gurkar, M. Foley, M. Schenone, X. Li, N.J. Tolliday, T.R. Golub, S.A. Carr, A.F. Shamji, A.M. Stern, A. Mandinova, S.L. Schreiber, S.W. Lee, Selective killing of cancer cells by a small molecule targeting the stress response to ROS, Nature, 475 (2011) 231-234.

[67] D. Trachootham, J. Alexandre, P. Huang, Targeting cancer cells by ROS-mediated mechanisms: a radical therapeutic approach?, Nature reviews. Drug discovery, 8 (2009) 579-591.

[68] G. Chen, F. Wang, D. Trachootham, P. Huang, Preferential killing of cancer cells with mitochondrial dysfunction by natural compounds, Mitochondrion, 10 (2010) 614-625.

[69] C. Cerella, F. Radogna, M. Dicato, M. Diederich, Natural compounds as regulators of the cancer cell metabolism, International journal of cell biology, 2013 (2013) 639401.
[70] R.A. Smith, M.P. Murphy, Mitochondria-targeted antioxidants as therapies, Discovery medicine, 11 (2011) 106-114.

[71] L. Galluzzi, G. Kroemer, Mitochondrial apoptosis without VDAC, Nature cell biology, 9 (2007) 487-489.

[72] J.E. Kokoszka, K.G. Waymire, S.E. Levy, J.E. Sligh, J. Cai, D.P. Jones, G.R. MacGregor, D.C. Wallace, The ADP/ATP translocator is not essential for the mitochondrial permeability transition pore, Nature, 427 (2004) 461-465.

[73] T. Yagi, B.B. Seo, E. Nakamaru-Ogiso, M. Marella, J. Barber-Singh, T. Yamashita, M.C. Kao, A. Matsuno-Yagi, Can a single subunit yeast NADH dehydrogenase (Ndi1) remedy diseases caused by respiratory complex I defects?, Rejuvenation research, 9 (2006) 191-197.

[74] J.J. Lemasters, Selective mitochondrial autophagy, or mitophagy, as a targeted defense against oxidative stress, mitochondrial dysfunction, and aging, Rejuvenation research, 8 (2005) 3-5.

[75] K. Degenhardt, R. Mathew, B. Beaudoin, K. Bray, D. Anderson, G. Chen, C. Mukherjee, Y. Shi, C. Gelinas, Y. Fan, D.A. Nelson, S. Jin, E. White, Autophagy promotes tumor cell survival and restricts necrosis, inflammation, and tumorigenesis, Cancer cell, 10 (2006) 51-64.

[76] S. Pavlides, I. Vera, R. Gandara, S. Sneddon, R.G. Pestell, I. Mercier, U.E. Martinez-Outschoorn, D. Whitaker-Menezes, A. Howell, F. Sotgia, M.P. Lisanti, Warburg meets autophagy: cancer-associated fibroblasts accelerate tumor growth and metastasis via oxidative stress, mitophagy, and aerobic glycolysis, Antioxidants \& redox signaling, 16 (2012) 1264-1284.

[77] J. H. Kim, H. Y. Kim, Y. K. Lee, Y.S. Yoon, W. G. Xu, J.K. Yoon, S.E. Choi, Y.G. Ko, M.J. Kim, S.J. Lee, H. J. Wang, G. Yoon, Involvement of mitophagy in oncogenic K-Ras-induced transformation: overcoming a cellular energy deficit from glucose deficiency, Autophagy, 7 (2011) 1187-1198.

[78] S. Yang, X. Wang, G. Contino, M. Liesa, E. Sahin, H. Ying, A. Bause, Y. Li, J.M. Stommel, G. Dell'antonio, J. Mautner, G. Tonon, M. Haigis, O.S. Shirihai, C. Doglioni, N. Bardeesy, A.C. Kimmelman, Pancreatic cancers require autophagy for tumor growth, Genes \& development, 25 (2011) 717-729.

[79] Z. J. Yang, C.E. Chee, S. Huang, F.A. Sinicrope, The role of autophagy in cancer: therapeutic implications, Molecular cancer therapeutics, 10 (2011) 1533-1541.

[80] R. Mathew, C.M. Karp, B. Beaudoin, N. Vuong, G. Chen, H.Y. Chen, K. Bray, A. Reddy, G. Bhanot, C. Gelinas, R.S. Dipaola, V. Karantza-Wadsworth, E. White, Autophagy suppresses tumorigenesis through elimination of p62, Cell, 137 (2009) 1062-1075.

[81] R. Scherz-Shouval, Z. Elazar, Regulation of autophagy by ROS: physiology and pathology, Trends in biochemical sciences, 36 (2011) 30-38. 